\title{
Characterization of a class of spatially interconnected systems (ladder circuits) using two-dimensional systems theory
}

\author{
Mohamed S. Boudellioua ${ }^{1} \cdot$ Krzysztof Galkowski $^{2}$ (D) Eric Rogers ${ }^{3}$
}

Received: 29 November 2018 / Revised: 29 March 2019 / Accepted: 1 April 2019 / Published online: 16 April 2019 (c) The Author(s) 2019

\begin{abstract}
This paper considers a class of spatially interconnected systems formed by ladder circuits using two-dimensional systems theory. The individual circuits in this class are described by hybrid (continuous/discrete) linear differential/difference equations in time (continuous) and spatial (discrete) variables and therefore have a two-dimensional systems structure. This paper shows that a ladder circuit model and models for 2-D dynamics have a well defined equivalence property and hence analysis tools can be transferred between them. Also the mechanism for transforming one to the other is established.
\end{abstract}

Keywords Spatially interconnected systems · Ladder circuit networks $\cdot$ Two-dimensional systems $\cdot$ Zero coprime system equivalence

\section{Introduction}

Spatially interconnected systems arise in a number of areas (D'Andrea and Dullerud 2003) (as one possible starting point for the literature) including electrical ladder networks/circuits, mechanical systems, composed, e.g, of masses and springs and heat transfer problems. For

Supported by Sultan Qaboos University (Oman) and by National Science Centre in Poland, Grant No. 2015/17/B/ST7/03703.

Krzysztof Galkowski

k.galkowski@issi.uz.zgora.pl

Mohamed S. Boudellioua

boudell@squ.edu.om

Eric Rogers

etar@ecs.soton.ac.uk

1 Department of Mathematics and Statistics, Sultan Qaboos University, Muscat, Oman

2 Institute of Control and Computation Engineering, University of Zielona Gora, Zielona Gora, Poland

3 School of Electronics and Computer Science, University of Southampton, Southampton SO17 1BJ, UK 


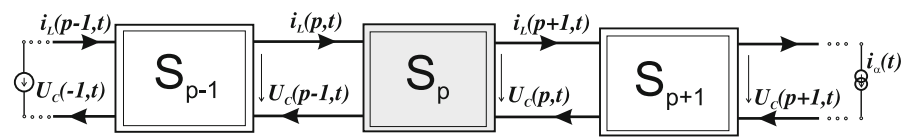

(a)

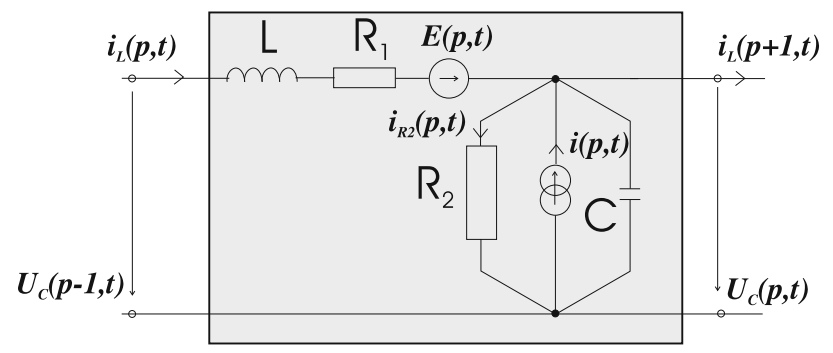

(b)

Fig. 1 Ladder circuit as a spatially interconnected system

these systems, time and spatial dynamics can arise. Hence they can be treated as two dimensional, or 2-D, systems, which is the setting used in this paper.

In many cases, these systems are composed of a finite number of blocks or cells. Hence, for analysis, there is the option to embed the spatial dynamics into the system variables using a form of lifting based on the discrete (finite) spatial variable and obtain a system where time is the independent variable, referred to as a 1D system in some of the 2-D systems literature, see, e.g. Sulikowski et al. (2015). One drawback of this approach, for systems composed of a large number of cells, is the need to compute with matrices that have very large dimensions and hence possible computational issues. The 2-D systems setting does not involve increasing the dimensions of the matrices used to represent the dynamics.

As one class, this paper considers electrical ladder networks or circuits, which are formed as a chain of blocks/cells that are identical in their structure. The passive parts of these cells are realized by longitudinal and transversal resistances, reactances, or, in general, impedances and the active parts by the addition of autonomous or controlled current and voltage sources. Application areas for ladder circuits include filter analysis and design, the modeling of delay lines and equivalent circuits for transmission lines, chains of transmission gates or long wire interconnections, e.g. Alioto et al. (2004), the approximation of distributed parameter systems, e.g. Schanbacher (1989) and in the simulation of physical systems. A representative example is shown in Fig. 1, where the upper diagram shows the overall system structure and the bottom the structure of an individual cell.

The dynamics of 2-D systems are characterized by information propagation in two independent directions and the independent variables can both be discrete, both continuous or mixed, i.e. one continuous and one discrete. Spatially interconnected systems, including ladder circuits, can be considered as a class of 2-D systems where the independent variables are the time, which can be continuous or discrete and the node/cell number, which has to be discrete. However, left-right and right-left dependence between neighboring cells are often needed. Such systems are not causal along the spatial axis, i.e. along the node/cell direction. Hence, they cannot be modeled by the standard and extensively investigated 2-D (Roesser 1975a) and (Fornasini and Marchesini 1976) state-space models. Also the well known stability analysis methods for these systems are not applicable. 
Repetitive processes are another class of 2-D dynamics that arise in the modeling of physical systems. These processes operate over a finite time duration and the 2-D structure arises as repeated passes are made through the dynamics and the output on any pass explicitly contributes to the dynamics produced on the next one. Background on these processes and the control problems they pose can be found in Rogers et al. (2007).

The links between linear repetitive processes and the Roesser and Fornasini-Marchesini state-space models has been investigated in previous research. For example, in Galkowski et al. (1998) the conditions for local controllability of linear repetitive processes have been derived via their transformation into the singular 2-D Roesser or Fornasini-Marchesini form and in Galkowski et al. (1999), building on previous work cited in this reference, the equivalence of their stability properties was investigated. This previous research established stability tests can, in some but not all, cases be interchanged. This is true for one class of repetitive processes but other classes can exhibit dynamics that have no Roesser or Fornasini-Marchesini state-space model representation. More recent work in this general area includes (Boudellioua et al. 2016, 2017; Galkowski et al. 2017) where new results on system equivalence are reported.

It is to be expected that at least some of these results would apply to spatially interconnected systems whose dynamics are written as 2-D linear systems. This is the motivation for the current paper where system equivalence between the state-space models of ladder circuits and those for the singular 2-D Roesser or Fornasini-Marchesini systems. The problem of reducing by equivalence a general 2-D polynomial matrix to the singular Roesser form has been considered previously, e.g. Pugh et al. (2005) developed a method for reducing a general 2-D polynomial matrix to such a form. Their method uses a two-step algorithm which is then adapted to the case of a general polynomial system matrix. In this paper, a direct method is developed to reduce a 2-D polynomial system matrix arising from a class of ladder circuit systems to an equivalent singular 2-D Fornasini-Marchesini or Roesser state-space model. The transformation used is shown to be zero coprime system equivalence of the two system matrices. This type of equivalence has previously been considered in the literature, e.g. Levy (1981), Johnson (1993), Boudellioua (2012), Pugh et al. (1996) and Pugh et al. (1998).

\section{State-space models and associated transfer functions}

In this paper, the general problem considered is the equivalence between ladder circuits and commonly used Roesser and Fornasini-Marchesini models for 2-D linear systems. In the remainder of this section, the relevant state-space models are introduced.

\subsection{Ladder circuits}

Applying Kirchhoff's laws, the dynamics of ladder circuits, see Fig. 2 for the configuration and layout, can be written in the form of a 2-D differential-discrete linear systems state-space model, see Sulikowski et al. (2015), where the independent variables are time and the node numbers $p=0,1, \ldots, \alpha-1$. The resulting model is

$$
\begin{aligned}
\frac{d}{d t} x(p, t)= & \mathcal{A}_{1} x(p-1, t)+\mathcal{A}_{2} x(p, t)+\mathcal{A}_{3} x(p+1, t) \\
& +\mathcal{B} u(p, t)
\end{aligned}
$$




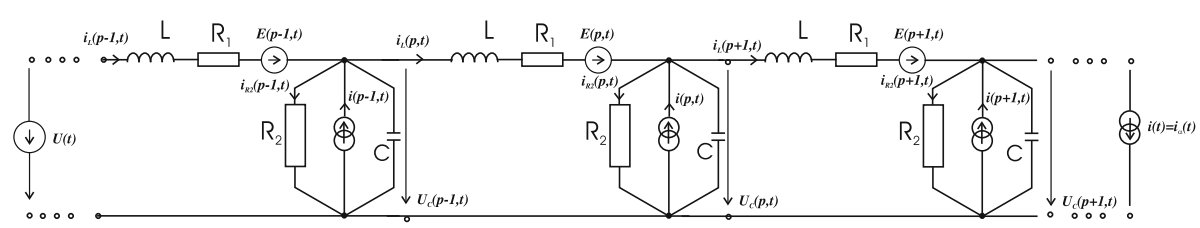

Fig. 2 A ladder chain

where $x(p, t)$ and $u(p, t)$, respectively, denote the state and input vectors. In this paper, the particular case of an active ladder circuit of the from of Fig. 1 is considered, which for onward analysis is considered in the form of Fig. 2, where the controlled sources $i(p, t)=$ $\gamma U_{c}(p-1, t)$ and $E(p, t)=u(p, t)$ have been added to the nodes as possible control input variables but could also be an intrinsic part of a particular circuit.

In this paper, the state vector for node $p$ in Fig. 2 is defined as

$$
x(p, t)=\left[\begin{array}{c}
U_{C}(p, t) \\
i_{L}(p, t)
\end{array}\right]
$$

and then the matrices in (1) are

$$
\begin{aligned}
\mathcal{A}_{1} & =\left[\begin{array}{cc}
\frac{\gamma}{C} & 0 \\
\frac{1}{L} & 0
\end{array}\right], \quad \mathcal{A}_{2}=\left[\begin{array}{cc}
-\frac{1}{R_{2} C} & \frac{1}{C} \\
-\frac{1}{L} & -\frac{R_{1}}{L}
\end{array}\right], \quad \mathcal{A}_{3}=\left[\begin{array}{cc}
0 & -\frac{1}{C} \\
0 & 0
\end{array}\right], \\
\mathcal{B} & =\left[\begin{array}{c}
0 \\
-\frac{1}{L}
\end{array}\right] .
\end{aligned}
$$

The output equation is

$$
y(p, t)=\mathcal{C} x(p, t),
$$

where the matrix $\mathcal{C}$ depends on which signals are chosen as output variables. To complete the description, the following boundary conditions are assumed

$$
\begin{aligned}
& x(-1, t)=\left[\begin{array}{c}
U(t) \\
0
\end{array}\right], x(\alpha, t)=\left[\begin{array}{c}
0 \\
i(t)
\end{array}\right], \\
& x(p, 0)=0,0 \leq p \leq \alpha-1 .
\end{aligned}
$$

Introduce the following differential and discrete, respectively, shift operators

$$
z_{1} x(p, t)=\frac{d x(p, t)}{d t}, \quad z_{2} x(p, t)=x(p+1, t) .
$$

Then the resulting transfer-function matrix under zero boundary conditions is

$$
G_{L C}\left(z_{1}, z_{2}\right)=\mathcal{C}\left(z_{1} I_{n}-z_{2} \mathcal{A}_{3}-\mathcal{A}_{2}-z_{2}^{-1} \mathcal{A}_{1}\right)^{-1} \mathcal{B}
$$

provided the inverse of the matrix $z_{1} I_{n}-z_{2} \mathcal{A}_{3}-\mathcal{A}_{2}-z_{2}^{-1} \mathcal{A}_{1}$ exists.

\subsection{2-D singular Fornasini-Marchesini model}

A singular version of the 2-D Fornasini-Marchesini state-space model (Fornasini and Marchesini 1976) for discrete linear dynamics is

$$
\operatorname{Ex}(i+1, j+1)=A_{1} x(i+1, j)+A_{2} x(i, j+1)
$$




$$
\begin{aligned}
& +A_{0} x(i, j)+B u(i, j) \\
y(i, j)= & C x(i, j)+D u(i, j),
\end{aligned}
$$

where $x(i, j)$ is the state vector, $u(i, j)$ is the input vector, $y(i, j)$ is the output vector, $E, A_{0}$, $A_{1}, A_{2}, B, C$ and $D$ are constant real matrices of compatible dimensions and $E$ is a singular matrix. In this paper, the model (1) is continuous in time $t$ and therefore the differential version of singular 2-D Fornasini-Marchesini state-space model, is required, i.e.

$$
\begin{aligned}
\mathbf{E} \frac{d \mathbf{x}(p+1, t)}{d t}= & \mathbf{A}_{1} \frac{d \mathbf{x}(p, t)}{d t}+\mathbf{A}_{2} \mathbf{x}(p+1, t) \\
& +\mathbf{A}_{0} \mathbf{x}(p, t)+\mathbf{B u}(p, t), \\
\mathbf{y}(p, t)= & \mathbf{C x}(p, t)+\mathbf{D u}(p, t),
\end{aligned}
$$

where $\mathbf{x}(p, t)$ is the state vector, $\mathbf{u}(p, t)$ is the input vector, $\mathbf{y}(p, t)$ is the output vector, $p$ denotes the node number and the matrices are as in (1). If $E$ is nonsingular in either model then the standard (or nonsingular) model is obtained.

Note 1 In this paper differential dynamics are considered. If, however, the system is sampled then the analysis still applies with the shift operator $z_{1}$ in (6) defined as a forward shift operator in $t$.

For systems described by (9), using the operators in (6) gives the transfer-function matrix for zero boundary conditions

$$
G_{D S F M}\left(z_{1}, z_{2}\right)=\mathbf{C}\left(z_{1} z_{2} \mathbf{E}-z_{1} \mathbf{A}_{1}-z_{2} \mathbf{A}_{2}-\mathbf{A}_{0}\right)^{-1} \mathbf{B}+\mathbf{D} .
$$

\subsection{2-D singular Roesser model}

A discrete singular Roesser state-space model (1975a) is given by

$$
\begin{aligned}
E\left[\begin{array}{l}
x^{h}(i+1, j) \\
x^{v}(i, j+1)
\end{array}\right] & =\left[\begin{array}{ll}
A_{11} & A_{12} \\
A_{21} & A_{22}
\end{array}\right]\left[\begin{array}{l}
x^{h}(i, j) \\
x^{v}(i, j)
\end{array}\right]+\left[\begin{array}{l}
B_{1} \\
B_{2}
\end{array}\right] u(i, j), \\
y(i, j) & =\left[\begin{array}{ll}
C_{1} & C_{2}
\end{array}\right]\left[\begin{array}{l}
x^{h}(i, j) \\
x^{v}(i, j)
\end{array}\right]+D u(i, j),
\end{aligned}
$$

where $x^{h}(i, j) \in \mathbb{R}^{n_{1}}$ is the horizontal state vector, $x^{v}(i, j) \in \mathbb{R}^{n_{2}}$, is the vertical state vector, $y(i, j) \in \mathbb{R}^{m}$ is the output vector, $u(i, j) \in \mathbb{R}^{l}$ is the input vector and the matrix $E$ is square and singular. In this model, static in both directions $i$ and $j$ links between sub-vectors are allowed. If $E$ is nonsingular then (as in the Fornasini-Marchesini model) the standard model is obtained. The boundary conditions are $x^{h}(0, j)=f(j), j \geq 0$ and $x^{v}(i, 0)=d(i), i \geq 0$, where the $n_{1} \times 1$ vector $f(j)$ and the $n_{2} \times 1$ vector $d(i)$ have known constant entries.

This paper requires the differential Roesser model given by

$$
\begin{aligned}
E\left[\begin{array}{c}
\frac{d x^{h}(p, t)}{d t} \\
x^{v}(p+1, t)
\end{array}\right] & =\left[\begin{array}{ll}
A_{11} & A_{12} \\
A_{21} & A_{22}
\end{array}\right]\left[\begin{array}{l}
x^{h}(p, t) \\
x^{v}(p, t)
\end{array}\right]+\left[\begin{array}{l}
B_{1} \\
B_{2}
\end{array}\right] u(p, t), \\
y(p, t) & =\left[\begin{array}{ll}
C_{1} & C_{2}
\end{array}\right]\left[\begin{array}{l}
x^{h}(p, t) \\
x^{v}(p, t)
\end{array}\right]+D u(p, t) .
\end{aligned}
$$

Using the shift operators $z_{1}$ and $z_{2}$ and assuming zero boundary conditions and that the matrix $z_{1} E_{1}+z_{2} E_{2}-A$ is invertible, the transfer-function matrix corresponding to (12) is

$$
G_{D S R}\left(z_{1}, z_{2}\right)=C\left(z_{1} E_{1}+z_{2} E_{2}-A\right)^{-1} B+D,
$$


where

$$
\begin{aligned}
E & =\left[\begin{array}{ll}
E_{11} & E_{12} \\
E_{21} & E_{22}
\end{array}\right], E_{1}=\left[\begin{array}{ll}
E_{11} & 0 \\
E_{21} & 0
\end{array}\right], E_{2}=\left[\begin{array}{ll}
0 & E_{12} \\
0 & E_{22}
\end{array}\right], A=\left[\begin{array}{ll}
A_{11} & A_{12} \\
A_{21} & A_{22}
\end{array}\right], \\
B^{T} & =\left[\begin{array}{ll}
B_{1}^{T} & B_{2}^{T}
\end{array}\right], C=\left[\begin{array}{ll}
C_{1} & C_{2}
\end{array}\right] .
\end{aligned}
$$

\section{Polynomial system matrix descriptions and system equivalence}

One general description of a 2-D linear system, in common with (Rosenbrock 1970) for the 1-D linear systems case, is the polynomial form

$$
\begin{aligned}
T\left(z_{1}, z_{2}\right) x & =U\left(z_{1}, z_{2}\right) u, \\
y & =V\left(z_{1}, z_{2}\right) x+W\left(z_{1}, z_{2}\right) u,
\end{aligned}
$$

where $x \in \mathbb{R}^{n}$ is the state vector, $u \in \mathbb{R}^{l}$ is the input vector and $y \in \mathbb{R}^{m}$ is the output vector, $T, U, V$ and $W$ are polynomial matrices with elements in $\mathbb{R}\left[z_{1}, z_{2}\right]$ of dimensions $n \times n, n \times l, m \times n$ and $m \times l$, respectively. The meaning of the operators $z_{1}$ and $z_{2}$ depend on the case considered as detailed in the previous section.

A system described by (14) can be rewritten as

$$
P\left(z_{1}, z_{2}\right)\left[\begin{array}{r}
x \\
-u
\end{array}\right]=\left[\begin{array}{r}
0 \\
-y
\end{array}\right],
$$

where

$$
P\left(z_{1}, z_{2}\right)=\left[\begin{array}{rr}
T\left(z_{1}, z_{2}\right) & U\left(z_{1}, z_{2}\right) \\
-V\left(z_{1}, z_{2}\right) & W\left(z_{1}, z_{2}\right)
\end{array}\right] .
$$

Assuming that $T\left(z_{1}, z_{2}\right)$ is invertible and the system matrix in (16) is regular, the associated transfer-function matrix is

$$
G\left(z_{1}, z_{2}\right)=V\left(z_{1}, z_{2}\right) T^{-1}\left(z_{1}, z_{2}\right) U\left(z_{1}, z_{2}\right)+W\left(z_{1}, z_{2}\right) .
$$

Using $P\left(z_{1}, z_{2}\right)$, the equivalence between systems can be studied, where numerous forms of this property are known in $n$-D systems. One of these for 2-D system matrices is zero coprimeness, see, e.g. Levy (1981), and Johnson (1993). This equivalence may be viewed as an extension of Fuhrmann's strict system equivalence (Fuhrmann 1977) from 1-D to 2-D systems and is defined as follows.

Definition 1 Let $\mathbb{P}(m, l)$ denote the class of $(n+m) \times(n+l)$ with polynomial system matrices in $z_{1}$ and $z_{2}$ with real coefficients. Two polynomial system matrices $P_{1}\left(z_{1}, z_{2}\right)$ and $P_{2}\left(z_{1}, z_{2}\right) \in \mathbb{P}(m, l)$, are said to be zero coprime system equivalent if they satisfy

$$
\begin{aligned}
& \underbrace{\left[\begin{array}{cc}
M\left(z_{1}, z_{2}\right) & 0 \\
X\left(z_{1}, z_{2}\right) & I_{m}
\end{array}\right]}_{S_{1}\left(z_{1}, z_{2}\right)} \underbrace{\left[\begin{array}{cc}
T_{1}\left(z_{1}, z_{2}\right) & U_{1}\left(z_{1}, z_{2}\right) \\
-V_{1}\left(z_{1}, z_{2}\right) & W_{1}\left(z_{1}, z_{2}\right)
\end{array}\right]}_{P_{1}\left(z_{1}, z_{2}\right)} \\
& =\underbrace{\left[\begin{array}{cc}
T_{2}\left(z_{1}, z_{2}\right) & U_{2}\left(z_{1}, z_{2}\right) \\
-V_{2}\left(z_{1}, z_{2}\right) & W_{2}\left(z_{1}, z_{2}\right)
\end{array}\right]}_{P_{2}\left(z_{1}, z_{2}\right)} \underbrace{\left[\begin{array}{cc}
N\left(z_{1}, z_{2}\right) & Y\left(z_{1}, z_{2}\right) \\
0 & I_{l}
\end{array}\right]}_{S_{2}\left(z_{1}, z_{2}\right)},
\end{aligned}
$$

where $P_{1}\left(z_{1}, z_{2}\right), S_{2}\left(z_{1}, z_{2}\right)$ are zero right coprime, $P_{2}\left(z_{1}, z_{2}\right), S_{1}\left(z_{1}, z_{2}\right)$ are zero left coprime and $M\left(z_{1}, z_{2}\right), N\left(z_{1}, z_{2}\right), X\left(z_{1}, z_{2}\right)$ and $Y\left(z_{1}, z_{2}\right)$ are polynomial matrices of compatible dimensions. 
The system matrix can be used to study critical systems properties such as controllability, observability and stability. In the case of 2-D linear systems, the zero structure of their associated system matrices, see, e.g. Zerz (2000), Johnson (1993), Levy (1981), Pugh et al. (1998), and Pugh et al. (1996), is of particular interest. One result is the following.

Lemma 1 (Johnson 1993) Zero coprime system equivalence preserves the transfer-function matrix and the zero structure of the matrices

$$
T_{i}\left(z_{1}, z_{2}\right), P_{i}\left(z_{1}, z_{2}\right),\left[T_{i}\left(z_{1}, z_{2}\right) U_{i}\left(z_{1}, z_{2}\right)\right],\left[\begin{array}{r}
T_{i}\left(z_{1}, z_{2}\right) \\
-V_{i}\left(z_{1}, z_{2}\right)
\end{array}\right], i=1,2 .
$$

The system matrix associated with the ladder circuit of (1) and (4) is

$$
P_{L C}\left(z_{1}, z_{2}\right)=\left[\begin{array}{c|c}
z_{1} I_{n}-z_{2} \mathcal{A}_{3}-\mathcal{A}_{2}-z_{2}^{-1} \mathcal{A} & \mathcal{B} \\
\hline-\mathcal{C} & 0_{m, l}
\end{array}\right] .
$$

An alternative description of (1) and (4) is obtained by forward shifting the node number in the state equations, which corresponds to multiplication by the shift operator $z_{2}$. This corresponds to multiplying the system matrix $P_{L C}$ in (19) from the left by the following matrix that preserves the transfer-function matrix

$$
\left[\begin{array}{c|c}
z_{2} I_{n} & 0 \\
\hline 0 & I_{l}
\end{array}\right]
$$

resulting in the polynomial system matrix

$$
\begin{aligned}
\tilde{P}_{L C}\left(z_{1}, z_{2}\right) & =\left[\begin{array}{c|c}
z_{1} z_{2} I_{n}-z_{2}^{2} \mathcal{A}_{3}-z_{2} \mathcal{A}_{2}-\mathcal{A} & z_{2} \mathcal{B} \\
\hline-\mathcal{C} & 0_{m, l}
\end{array}\right] \\
& =\left[\begin{array}{ll}
-\mathcal{A} & 0 \\
-\mathcal{C} & 0
\end{array}\right]+\left[\begin{array}{ll}
I & 0 \\
0 & 0
\end{array}\right] z_{1} z_{2}+\left[\begin{array}{cc}
-\mathcal{A}_{2} & \mathcal{B} \\
0 & 0
\end{array}\right] z_{2}+\left[\begin{array}{cc}
-\mathcal{A}_{3} & 0 \\
0 & 0
\end{array}\right] z_{2}^{2} .
\end{aligned}
$$

A system described by (9) can be written as

$$
\begin{gathered}
P_{a}\left(z_{1}, z_{2}\right)\left[\begin{array}{r}
\mathbf{x} \\
-\mathbf{u}
\end{array}\right] \\
=\left[\begin{array}{r}
0 \\
-\mathbf{y}
\end{array}\right],
\end{gathered}
$$

with polynomial system matrix

$$
P_{a}\left(z_{1}, z_{2}\right)=\left[\begin{array}{c|c}
z_{1} z_{2} \mathbf{E}-z_{1} \mathbf{A}_{1}-z_{2} \mathbf{A}_{2}-\mathbf{A}_{0} & \mathbf{B} \\
\hline-\mathbf{C} & \mathbf{D}
\end{array}\right] .
$$

Similarly a system described by (12) can be written as

$$
P_{b}\left(z_{1}, z_{2}\right)\left[\begin{array}{r}
x_{h} \\
x_{v} \\
-u
\end{array}\right]=\left[\begin{array}{r}
0 \\
0 \\
-y
\end{array}\right]
$$

with polynomial system matrix

$$
P_{b}\left(z_{1}, z_{2}\right)=\left[\begin{array}{c|c}
z_{1} E_{1}+z_{2} E_{2}-A & B \\
\hline-C & D
\end{array}\right] .
$$

Next, conditions under which the polynomial system matrix of the ladder circuit is equivalent to (9) are established. 


\section{Transformation of the ladder circuit model to a differential Fornasini-Marchesini model}

The polynomial system matrix of (20) can be written as

$$
\tilde{P}_{L C}\left(z_{1}, z_{2}\right)=\tilde{P}_{0,0}+\tilde{P}_{1,1} z_{1} z_{2}+\tilde{P}_{0,1} z_{2}+\tilde{P}_{0,2} z_{2}^{2} .
$$

Also introduce the matrices

$$
\begin{aligned}
\tilde{E} & =\left[\begin{array}{cc}
0_{n+l, n+l} & 0_{n+l, n+l} \\
0_{n+m, n+l} & \tilde{P}_{1,1}
\end{array}\right], \\
\tilde{A}_{0} & =\left[\begin{array}{cc}
-I_{n+l} & 0_{n+l, n+l} \\
0_{n+m, n+l} & -\tilde{P}_{0,0}
\end{array}\right], \\
\tilde{A}_{1} & =0, \quad \tilde{A}_{2}=\left[\begin{array}{cc}
0_{n+l, n+l} & I_{n+l} \\
-\tilde{P}_{0,2} & -\tilde{P}_{0,1}
\end{array}\right], \\
Y_{m} & =\left[\begin{array}{c}
0_{2 n+l, m} \\
I_{m}
\end{array}\right], Z_{l}=\left[\begin{array}{ll}
0_{l, 2 n+l} & I_{l}
\end{array}\right]
\end{aligned}
$$

and hence the polynomial system matrix

$$
P_{D S F M}\left(z_{1}, z_{2}\right) \equiv\left[\begin{array}{cc}
T_{S F M} & U_{S F M} \\
-V_{S F M} & 0_{m, l}
\end{array}\right]=\left[\begin{array}{cc|c}
z_{1} z_{2} \tilde{E}-z_{2} \tilde{A}_{2}-\tilde{A}_{0} & Y_{m} & 0 \\
-Z_{l} & 0_{l, m} & I_{l} \\
\hline 0_{m, n+l} & -I_{m} & 0_{m, l}
\end{array}\right] .
$$

This matrix corresponds to that of (22) with

$$
\mathbf{E}=\left[\begin{array}{cc}
\tilde{E} & 0 \\
0 & 0
\end{array}\right], \mathbf{A}_{0}=\left[\begin{array}{cc}
\tilde{A}_{0} & -Y_{m} \\
Z_{l} & 0
\end{array}\right], \mathbf{A}_{2}=\left[\begin{array}{cc}
\tilde{A}_{2} & 0 \\
0 & 0
\end{array}\right], \quad \mathbf{A}_{1}=0 .
$$

Theorem 1 The polynomial system matrices (27) and (20) are zero coprime equivalent i.e.

$$
S_{1} \tilde{P}_{L C}=P_{D S F M} S_{2},
$$

where

$$
S_{1}=\left[\begin{array}{c|c}
0_{n+l, n} & 0_{n+l, m} \\
I_{n} & 0_{n, m} \\
0_{m+l, n} & 0_{m+l, m} \\
\hline 0_{m, n} & I_{m}
\end{array}\right], \quad S_{2}=\left[\begin{array}{c|c}
z_{2} I_{n} & 0_{n, l} \\
0_{l, n} & z_{2} I_{l} \\
I_{n} & 0_{n, l} \\
0_{l, n} & I_{l} \\
\mathcal{C} & 0_{m, l} \\
\hline 0_{l, n} & I_{l}
\end{array}\right] .
$$

Proof The matrix of (27) can be written as

$$
\left[\begin{array}{ccc|c}
I_{n+l} & -z_{2} I_{n+l} & 0 & 0 \\
z_{2} \tilde{P}_{0,2} & z_{1} z_{2} \tilde{P}_{1,1}+z_{1} \tilde{P}_{0,1}+z_{2} \tilde{P}_{0,1}+\tilde{P}_{0,0} & \mathcal{F}_{m}^{T} & 0 \\
0 & -\mathcal{F}_{l} & 0 & I_{l} \\
\hline 0 & 0 & -I_{m} & 0
\end{array}\right],
$$

where $\mathcal{F}_{k}=\left[\begin{array}{ll}0_{k, n} & I_{k}\end{array}\right]$ and hence (29) holds, i.e.

$$
S_{1} \tilde{P}_{L C}=P_{D S F M} S_{2}=\left[\begin{array}{cc}
0_{n+l, n} & 0_{n+l, l} \\
z_{1} z_{2} I_{n}-z_{2}^{2} \mathcal{A}_{3}-z_{2} \mathcal{A}_{2}-\mathcal{A} & z_{2} \mathcal{B} \\
0_{m+l, n} & 0_{m+l, l} \\
-\mathcal{C} & 0_{m, l}
\end{array}\right] .
$$


Finally, zero right coprimeness of the two matrices follows since the matrix

$$
\left[\begin{array}{c}
\tilde{P}_{L C} \\
S_{2}
\end{array}\right]=\left[\begin{array}{cc}
z_{1} z_{2} I_{n}-z_{2}^{2} \mathcal{A}_{3}-z_{2} \mathcal{A}_{2}-\mathcal{A} & \tilde{z}_{2} \mathcal{B} \\
-\mathcal{C} & 0_{m, l} \\
\hline z_{2} I_{n} & 0_{l, l} \\
0 & z_{2} I_{n} \\
I_{n} & 0_{n, l} \\
0_{l, n} & I_{l} \\
\mathcal{C} & 0_{m, l} \\
0_{l, n} & I_{l}
\end{array}\right],
$$

contains a minor of order $n+l$ (the identity submatrix). Similarly, zero left coprimeness of the two matrices follows immediately since

$$
\left[\begin{array}{ll}
P_{D S F M} & S_{1}
\end{array}\right]=\left[\begin{array}{ll}
X_{11} & X_{12} \\
X_{21} & X_{22}
\end{array}\right]
$$

where



and

$$
\begin{aligned}
& X_{12}=\left[\begin{array}{cc}
0 & 0 \\
I_{n} & 0 \\
0 & 0
\end{array}\right] \text {, } \\
& X_{21}=\left[\begin{array}{llll}
0 & 0 & -I_{m} & 0
\end{array}\right], \quad X_{22}=\left[\begin{array}{ll}
0 & I_{m}
\end{array}\right],
\end{aligned}
$$

has a highest order minor equal to 1 obtained by deleting the columns $n+l+1, \ldots, 2(n+l)$ from the matrix in (34).

\section{Equivalence of the ladder circuit and the differential singular Roesser model}

The polynomial system matrix (20) can be written as

$$
\tilde{P}_{L C}\left(z_{1}, z_{2}\right)=\tilde{P}_{0,0}+\tilde{P}_{1,1} z_{1} z_{2}+\tilde{P}_{0,1} z_{2}+\tilde{P}_{0,2} z_{2}^{2} .
$$

Introduce the matrices

$$
\begin{gathered}
\tilde{E}=\left[\begin{array}{ccc}
0_{n+l, n+l} & 0_{n+l, n+l} & 0 \\
0_{n+m, n+l} & \tilde{P}_{1,1} & 0 \\
0 & 0 & 0
\end{array}\right], \tilde{A}_{0}=\left[\begin{array}{ccc}
-I_{n+l} & 0_{n+l, n+l} & 0 \\
0_{n+m, n+l} & -\tilde{P}_{0,0} & -\mathcal{F}_{m, n+m}^{T} \\
0 & \mathcal{F}_{l, n+l} & 0
\end{array}\right], \\
\tilde{A}_{2}=\left[\begin{array}{ccc}
0_{n+l, n+l} & 0_{n+l, n+l} & 0 \\
-\tilde{P}_{0,2} & -\tilde{P}_{0,1} & 0 \\
0 & 0 & 0
\end{array}\right] .
\end{gathered}
$$

Then the polynomial system matrix in singular differential Roesser form is given by 


$$
\begin{aligned}
& P_{S R}\left(z_{1}, z_{2}\right) \equiv\left[\begin{array}{cc}
T_{S R} & U_{S R} \\
-V_{S R} & 0_{m, l}
\end{array}\right] \\
& =\left[\begin{array}{cccc|c}
I_{2(n+l)+m} & -z_{1} I_{2(n+l)+m} & 0_{2(n+l)+l, l} & 0_{2(n+l)+l, m} & 0_{2(n+l)+l, l} \\
z_{2} \tilde{E} & -z_{2} \tilde{A}_{2}-\tilde{A}_{0} & \mathcal{F}_{2(n+l)+m, l}^{T} & 0_{2(n+l)+m, m} & 0_{2(n+l)+m, l} \\
0_{m, 2(n+l)+m} & -\mathcal{F}_{m, 2(n+l)+m} & 0_{m, l} & I_{m} & 0_{m, l} \\
0_{l, 2(n+l)+m} & 0_{l, 2(n+l)+m} & -I_{l} & 0_{l, m} & I_{l} \\
\hline 0_{m, 2(n+l)+m} & 0_{m, 2(n+l)+m} & 0_{m, l} & -I_{m} & 0_{m, l}
\end{array}\right],
\end{aligned}
$$

where $\mathcal{F}_{r, k}=\left[\begin{array}{ll}0_{r, k-r} & I_{r}\end{array}\right]$.

Theorem 2 The system matrices (37) and (20) are zero coprime system equivalent, i.e.

$$
S_{1} \tilde{P}_{L C}=P_{S R} S_{2},
$$

where

$$
S_{1}=\left[\begin{array}{c|c}
0 & 0 \\
I_{t} & 0 \\
0 & 0 \\
0 & 0 \\
\hline 0 & I_{m}
\end{array}\right], \quad S_{2}=\left[\begin{array}{cc|c}
z_{1} z_{2} I_{n} & 0 \\
0 & z_{1} z_{2} I_{l} \\
z_{1} I_{n} & 0 \\
0 & z_{1} I_{l} \\
z_{1} \mathcal{F}_{m, t} & 0 \\
z_{2} I_{n} & 0 \\
0 & z_{2} I_{l} \\
I_{n} & 0 \\
0 & I_{l} \\
\mathcal{F}_{m, n} & 0_{m, l} \\
0 & I_{l} \\
\mathcal{F}_{l, n} & 0 \\
\hline 0 & I_{l}
\end{array}\right] .
$$

Proof It can be verified that

$$
S_{1} \tilde{P}_{L C}=P_{S R} S_{2}=\left[\begin{array}{cc}
0 & 0 \\
\tilde{T}_{L C} & \tilde{U}_{L C} \\
0 & 0 \\
0 & 0 \\
\tilde{V}_{L C} & 0_{m, l}
\end{array}\right]
$$

and it remains to establish the zero coprimeness of the matrices, where zero right coprimeness of $\tilde{P}_{L C}$ and $S_{2}$ follows from the fact that the matrix $\left[\begin{array}{c}\tilde{P}_{L C} \\ S_{2}\end{array}\right]$ contains a highest order minor of order $n+l$ which is equal to 1 . The zero left coprimeness of $P_{S R}$ and $S_{1}$ follows since the matrix

$$
\left[\begin{array}{ll}
P_{S R} & S_{1}
\end{array}\right]=\left[\begin{array}{ccccc|cc}
I_{2(n+l)+m} & -z_{1} I_{2(n+l)+m} & 0 & 0 & 0 & 0 & 0 \\
z_{2} \tilde{E} & -z_{2} \tilde{A}_{2}-\tilde{A}_{0} & \mathcal{F}_{2(n+l)+m, l}^{T} & 0 & 0 & I_{2(n+l)+m} & 0 \\
0 & -\mathcal{F}_{m, 2(n+l)+m} & 0 & I_{m} & 0 & 0 & 0 \\
0 & 0 & -I_{l} & 0 & I_{l} & 0 & 0 \\
0 & 0 & 0 & -I_{m} & 0 & 0 & I_{m}
\end{array}\right],
$$

has a highest order minor of order $2[2(n+l)+l+m]+m$ which is equal to \pm 1 , obtained by deleting the second and third block columns of the matrix in (41). 


\section{Conclusions}

In this paper, an equivalent representation is obtained in the form of 2-D Fornasini-Marchesini and Roesser singular state-space models for a given system matrix arising from a hybrid linear ladder circuit system. The exact connections between the original system matrix with its corresponding 2-D singular forms have been developed and shown to be zero coprime system equivalence. Also the zero structure of the original polynomial system matrix is preserved, making it possible to analyze the polynomial system matrix in terms of its associated 2D singular form. Moreover, the transformation matrices include identity sub-matrices and this suggests that these transformations can be generated by finite sequences of elementary row/column operations together with trivial inflation/deflation of the polynomial system matrices. This area is the subject of ongoing research. Also the implications of these results in terms of the structure and design of control laws is also under investigation.

Acknowledgements This work has been supported by Sultan Qaboos University (Oman) and also by the National Science Centre in Poland, Grant No. 2015/17/B/ST7/03703.

Open Access This article is distributed under the terms of the Creative Commons Attribution 4.0 International License (http://creativecommons.org/licenses/by/4.0/), which permits unrestricted use, distribution, and reproduction in any medium, provided you give appropriate credit to the original author(s) and the source, provide a link to the Creative Commons license, and indicate if changes were made.

\section{References}

Alioto, M., Palumbo, G., \& Poli, M. (2004). Evaluation of energy consumption in RC ladder circuits driven by a ramp input. IEEE Transactions on Very Large Scale Integration (VLSI) Systems, 12(10), 1094-1107.

Boudellioua, M., Galkowski, K., \& Rogers, E. (2017). Reduction of discrete linear repetitive processes to nonsingular Roesser models via elementary operations. In IFAC 2017 World Congresss, Toulouse, France (p. 2017).

Boudellioua, M. S. (2012). Strict system equivalence of 2D linear discrete state space models. Journal of Control Science and Engineering, 2012(Art. ID 609276): 6 pages.

Boudellioua, M. S., Galkowski, K., \& Rogers, E. (2016). On the connection between discrete linear repetitive processes and 2-D discrete linear systems. In Multidimensional systems and signal processing, 11. https:// doi.org/10.1007/s11045-016-0454-8.

D’Andrea, R., \& Dullerud, G. (2003). Distributed control design for spatially interconnected systems. IEEE Transactions on Automatic Control, 48(9), 1478-1495.

Fornasini, E., \& Marchesini, G. (1976). State space realization theory of two-dimensional filters. IEEE Transactions on Automatc Control, AC-21(4), 484-492.

Fuhrmann, P. A. (1977). On strict system equivalence and similarity. International Journal Control, 25(1), $5-10$.

Galkowski, K., Boudellioua, M., Rogers, E. (2017). Reduction of wave linear repetitive processes to singular roesser model form. In The 23rd international conference on methods and models in automation and robotics (MMAR 2017), Miedzyzdroje, Poland.

Galkowski, K., Rogers, E., \& Owens, D. H. (1998). Matrix rank based tests for reachabiltiy/controllability of discrete linear repetitive processes. Linear Algebra and its Applications, 275-276, 201-224.

Galkowski, K., Rogers, E., \& Owens, D. H. (1999). New 2D models and a transition matrix for discrete linear repetitive processes. International Journal of Control, 72(5), 1365-1380.

Johnson, D. S. (1993). Coprimeness in multidimensional system theory and symbolic computation. Ph.D thesis, Loughborough University, UK.

Levy, B. C. (1981). 2-D polynomial and rational matrices and their applications for the modelling of 2-D dynamical systems. Ph.D. thesis, Stanford University, USA

Pugh, A. C., McInerney, S. J., Boudellioua, M. S., Johnson, D. S., \& Hayton, G. E. (1998). A transformation for 2-D linear systems and a generalization of a theorem of Rosenbrock. International Journal Control, 71(3), 491-503. 
Pugh, A. C., McInerney, S. J., \& El-Nabrawy, E. M. O. (2005). Equivalence and reduction of 2-D systems. IEEE Transactions on Circuits and Systems-II: Express Briefs, 52(5), 271-275.

Pugh, A. C., McInerney, S. J., Hou, M., \& Hayton, G. E. (1996) A transformation for 2-D systems and its invariants. In Proceedings of the 35th IEEE conference on decision and control, pp. 2157-2158, Kobe (Japan).

Roesser, R. P. (1975). A discrete state-space model for linear image processing. IEEE Transactions on Automatic Control, 20(1), 1-10.

Rogers, E., Galkowski, K., \& Owens, D. H. (2007). Control systems theory and applications for linear repetitive processes. Control and Information Sciences. Berlin, Heidelberg: Springer.

Rosenbrock, H. H. (1970). State space and multivariable theory. London, New York: Nelson-Wiley.

Schanbacher, T. (1989). Aspects of positivity in control theory. SIAM Journal on Control and Optimization, 27(3), 457-475.

Sulikowski, B., Galkowski, K., \& Kummert, A. (2015). Proportional plus integral control of ladder circuits modeled in the form of two-dimensional (2D) systems. Multidimesional Systems and Signal Processing, 26(1), 267-290.

Zerz, E. (2000). Topics in multidimensional linear systems theory. London: Springer.

Publisher's Note Springer Nature remains neutral with regard to jurisdictional claims in published maps and institutional affiliations.



Mohamed S. Boudellioua received a B.Eng. in mechanical engineering and an M.Sc. in control engineering from Bradford university in 1981 and 1982 respectively. He worked for Nottingham Trent Polytechnic, now Nottingham Trent university, as a research assistant/demonstrator from 1983 to 1986. He received a Ph.D. degree in applied mathematics in 1986 for a thesis on multidimensional systems theory. From 1987 to 1994 he was an associate professor at Skikda University (Algeria) and from 1994 to 2001 with Al-Ahsa College of Technology (Saudi Arabia). Since 2001 he has been with Sultan Qaboos University (Oman). His research interests include algebraic methods in systems theory and control in particular multidimensional systems.

Krzysztof Galkowski is Professor of Technical Sciences in University of Zielona Gora and also in Nicolaus Copernicus University in Torun, Poland. His major research interests lie in the areas of multidimensional (nD) systems and control theory and applications for repetitive processes, including application to iterative learning control with experimental validation. He has authored over 100 papers in leading peer reviewed journals and over 200 papers in the proceedings of major international conferences. He is an associate editor of IET Control Theory and Applications and a member of the editorial boards of the International Journal of Control and Multidimensional Systems and Signal Processing. He is the co-author/editor of 4 research monographs/books and also a guest editor of special issues in leading international journals, including the International Journal of Control, Multidimensional Systems and Signal Processing and the Asian Journal of Control. He is a co-founder of the international workshops on multidimensional systems (NDS). He is a visiting professor in the School of Electronics and Computer Science, University of Southampton, UK and was three times a Gerhard Mercator guest professor, funded by DFG, in The University of Wuppertal, Germany (09.2004-08.2005), (10.2006-09.2007) and (03.2011-02.2012). During the period 2011-2014 he was a Chair Professor in East China University of Science and Technology in Shanghai, China.

Eric Rogers was born in Northern Ireland and completed the undergraduate degree in Mechanical Engineering at Queen's University Belfast. After this he switched to control systems and was awarded the postgraduate masters and $\mathrm{Ph} . \mathrm{D}$. degrees in this subject by the University of Sheffield, UK. He is currently Professor of Control Systems Theory and Design, School of Electronics and Computer Science, University of Southampton, UK. His major research interests currently include multidimensional systems theory and applications, iterative learning control and applications, including application to robotic-assisted stroke 
rehabilitation with supporting clinical trials and smart rotor control for wind turbines and estimation and control of autonomous underwater vehicles with particular interests in long range autonomy and close sea bed following. He is currently Editor-in-Chief of the International Journal of Control and of Multidimensional Systems and Signal Processing. 